\title{
Evaluation of mitral regurgitation by an integrated 2D echocardiographic approach in patients undergoing transcatheter aortic valve replacement
}

\author{
R. Jansen ${ }^{1}$ - A. M. Wind ${ }^{1}$ - M. J. Cramer ${ }^{1}$ - F. Nijhoff ${ }^{1}$ - P. Agostoni ${ }^{1,2} \cdot$ F. Z. Ramjankhan ${ }^{3} \cdot$ W. J. Suyker ${ }^{3} \cdot$ P. R. Stella ${ }^{1}$. \\ S. A. J. Chamuleau ${ }^{1}$
}

Received: 21 November 2017 / Accepted: 2 March 2018 / Published online: 10 March 2018

(c) The Author(s) 2018. This article is an open access publication

\begin{abstract}
The purpose of this study was to evaluate mitral regurgitation (MR) severity in patients undergoing transcatheter aortic valve replacement (TAVR) by standardized assessment of two-dimensional (2D) transthoracic echocardiography (TTE) and 1-year echocardiographic and clinical outcomes. Pre- and post-procedural TTE's of patients undergoing TAVR between 2008 and 2014 were analyzed. MR was graded according to current guidelines with a systematic and integrated approach. Longitudinal echocardiographic and clinical results were analyzed. Regression analysis was performed for change in MR grade at follow-up, using pre-determined variables and confounders. Pre- and post-procedural TTE were available in 213 subjects. Significant MR was seen in 22\% at baseline and 15\% at follow-up; MR grade $\geq 3$ in $<10 \%$. Severity did not change in $61 \%$, and decreased in $20 \%$ of the patients. Overall, the prevalence of MR grades pre- and post TAVR was not significantly different, nor influenced by MR etiology or TAVR prosthesis type. However, higher MR grades and pacemaker absence at baseline, were independently correlated to more improvement of MR after TAVR. Regarding clinical outcomes, NYHA class improved in two-thirds of the patients, irrespective of the baseline MR grade. Overall survival was not significantly different amongst MR grades post-TAVR. MR grading using an systematic 2D echocardiographic approach in patients undergoing TAVR is feasible in clinical practice. Our data revealed a relatively frequent prevalence of significant MR (although grade $\geq 3$ was scarce), overall no change in the MR grade at 1 year follow-up, improvement of functional NYHA class, and no significant differences in long-term survival amongst the post-TAVR MR grades.
\end{abstract}

Keywords Mitral regurgitation $\cdot$ Transcatheter aortic valve replacement $\cdot 2 \mathrm{D}$ transthoracic echocardiography $\cdot$ Scoring index

\section{Introduction}

Electronic supplementary material The online version of this article (https://doi.org/10.1007/s10554-018-1328-y) contains supplementary material, which is available to authorized users.

S. A. J. Chamuleau

s.a.j.chamuleau@umcutrecht.nl

1 Department of Cardiology, University Medical Center Utrecht, Heidelberglaan 100, 3584 CX Utrecht, The Netherlands

2 Department of Cardiology, St. Antonius Hospital Nieuwegein, Nieuwegein, The Netherlands

3 Department of Cardiothoracic Surgery, University Medical Center Utrecht, Utrecht, The Netherlands
Aortic stenosis (AS) is the most frequent heart valve disease in Europe, followed by mitral regurgitation (MR) [1]. Both have become a major cause of morbidity and mortality among a growing and aging population [2, 3]. Especially in high risk patients surgical treatment is challenging. In response to this clinical challenge, percutaneous treatment options have rapidly evolved. The actual transcatheter aortic valve replacement (TAVR) rate has already increased to more than 100,000 replacements worldwide [4].

In addition to the individual burden of valve disease, significant AS is frequently associated with MR of varying degrees. The etiology is mostly functional due to left ventricular (LV) remodeling and increased afterload, but may also be organic from mitral annular calcification, or myxomatous 
and rheumatic degeneration. Mild MR is common in patients with severe AS (60-90\%) [5], although reported prevalence of significant MR disease varies between 19 and 33\% [6-12]. This variation may result from differences in MR grading. The importance of correct and consistent MR grading in patients with AS is related to previous studies in conventional aortic valve surgery, in which concomitant moderate MR has demonstrated to be an independent risk factor for mortality and morbidity, and may improve significantly in a selected group of patients avoiding unnecessary double valve surgery [13-15]. However, the predictive value of MR on clinical outcome after TAVR is still controversial, and discrepant results have been reported in terms of direction, magnitude and predictors of MR changes following TAVR $[11,12,16-19]$.

Two-dimensional (2D) transthoracic echocardiography (TTE) still is the principal examination in daily practice. Accurate MR grading is challenging, especially in patients with severe AS due to the high LV pressure afterload influencing qualitative echo parameters, and technical difficulties in determining the quantitative variables. Unfortunately assessment of concomitant MR in patients with AS is still based on visual color analysis of jet characteristics, whilst an integrated approach involving both quantitative and qualitative measurements is advisable [12, 20-22]. Studies that claim to use the guideline recommendations for MR grading neither give insight in the number of variables that could be determined, nor in the actual number of parameters that were evaluated [11, 16, 17].

Overall, systematic grading of MR severity may have important clinical implications in patients undergoing TAVR and thus the aim of our study was to (1) assess MR severity pre- and post TAVR by an integrated approach for $2 \mathrm{D}$ echo and evaluate grading in daily practice, (2) determine variables that are related to change in MR at 1 year post TAVR, and (3) evaluate clinical outcome after TAVR.

\section{Materials and method}

\section{Study population}

Figure 1 depicts the flowchart of the study design. Follow-up data of 354 patients that underwent TAVR in the University Medical Center of Utrecht (UMCU) between 2008 and 2014 were collected. We excluded 138 patients with incomplete
Fig. 1 Flowchart of the study design. TAVR transcatheter aortic valve replacement, $2 D$ two-dimensional, TTE transthoracic echocardiogram, $M R$ mitral regurgitation, ROSE-index mitral RegurgitatiOn Severity grading by an Easy-to-use index. $*$ ROSE-index score $=($ valve morphology*1)+(jet characteristics $* 2)+($ vena contracta $* 2)+($ systolic rever$\mathrm{sal} * 2)+($ LV dimensions $* 1)$

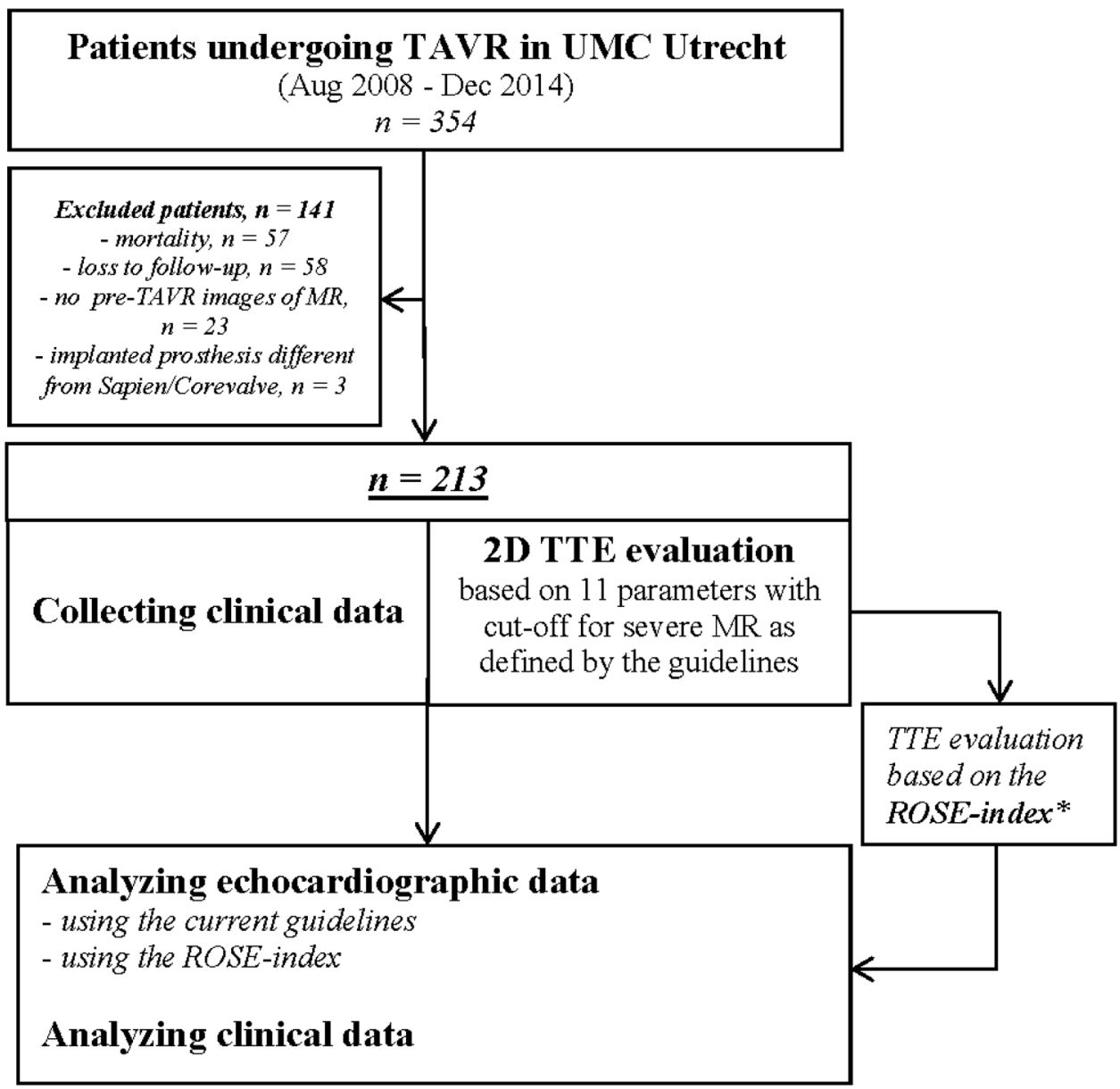


TTE data (57 died). Patients that underwent TAVR with an implanted prosthesis different from Edwards Sapien (Edwards Lifescience, Irvine, CA, USA), or Corevalve (3) (Medtronic, Minneapolis, MN, USA) were also excluded, leaving 213 subjects for retrospective echo analysis.

\section{Data collection}

Pre-, peri- and postoperative information was retrieved from the electronic patient record system of the UMCU. Echocardiographic examinations were collected from the hospital server where they were archived as video loops and freeze frames in a digital format (DICOM). Off line analysis was performed using Xcelera software. Systematic and standardized 2D TTE assessment was performed in all included subjects utilizing multiple parasternal and apical views. Echocardiography was performed by a member of an experienced team of sonographers, and supervised by one of the cardiologists with special interest for cardiac imaging. Based on the echocardiographic information each patient was classified as predominantly functional, organic or absence of MR. MR was considered to be organic in case of morphological abnormalities of the mitral apparatus. Valvular regurgitation caused by secondary changes due to abnormal ventricular size and deformation, or MR in absence of structural changes, was regarded as functional.

\section{Echocardiographic evaluation}

Echocardiographic measurements were obtained in accordance with the international guidelines, with focus on the European recommendations [20, 21, 23, 24]. All the analyzable parameters were systematically scored, focused on the recommended 11 echo parameters: mitral valve (MV) morphology, regurgitant jet characteristics, MV inflow (E-wave), ratio of the time-velocity integral (TVI) MV over the TVI left ventricular outflow tract (LVOT), mentioned as TVI MV/TVI LVOT, vena contracta (VC), effective regurgitant orifice (ERO), regurgitant volume, left atrial volume indexed (LAVI), LV dimensions, systolic reversal of the pulmonary vein flow, and systolic artery pulmonary pressure (SPAP). In Table 1 the definitions and cut-off values for severe MR are depicted. MV morphology resembled severe MR in case of flail leaflet, papillary muscle rupture, annular dilatation (end-diastolic diameter $>3.5 \mathrm{~cm}$ ), significant tethering of the valves, rheumatic etiology, presence of a cleft and/or (previous) endocarditis. More severe MR was to be expected in case regurgitation jet characteristics with presence of a swirling jet, jet reaching the left atrial posterior wall, and/ or jet to left atrial surface ratio of $>40 \%$ [20, 21, 23, 24]. Analysis of jet characteristics was done in multiple views. The VC was imaged in the parasternal long-axis or apical 4-chamber view and measured as the narrowest part of the
Table 1 Cut-off values for severe MR per parameter as defined by the current European guidelines [21, 25]

\begin{tabular}{ll}
\hline Parameter & $\begin{array}{l}\text { Cut-off values based on } \\
\text { the European guidelines }\end{array}$ \\
\hline $\begin{array}{l}\text { 1. Valve morphology } \\
\text { Annular dilatation }\end{array}$ & $3.5 \mathrm{~cm}$ \\
2. Jet characteristics & - \\
3. VC & $0.70 \mathrm{~cm}$ \\
4. ERO & \\
Organic MR & $0.40 \mathrm{~cm}^{2}$ \\
Functional MR & $0.20 \mathrm{~cm}^{2}$ \\
5. Regurgitant volume & \\
Organic MR & $60 \mathrm{ml}$ \\
Functional MR & $30 \mathrm{ml}$ \\
MV inflow pattern & \\
6. TVI MV/TVI LVOT ratio & 1.4 \\
7. E-wave & $150 \mathrm{~cm} / \mathrm{s}$ \\
8. Systolic flow in pulmonary veins & Systolic reversal \\
9. SPAP & $50 \mathrm{mmHg}$ \\
10. LAVI & $60 \mathrm{ml} / \mathrm{m}^{2}$ \\
11. LV dimensions & \\
Organic MR (LVESD) & $4.5 \mathrm{~cm}$ a \\
Functional MR (LVEDD) & $6.5 \mathrm{~cm}$ \\
\hline
\end{tabular}

$V C$ vena contracta, ERO effective regurgitant orifice, $M R$ mitral regurgitation, $M V$ mitral valve, TVI time-velocity integral, $L V O T$ left ventricular outflow tract, SPAP systolic pulmonary artery pressure, $L A V I$ left atrial volume index, $L V$ left ventricular, $L V E S D$ left ventricular end systolic dimension, $L V E D D$ left ventricular diastolic dimension

${ }^{\mathrm{a}} 4.0 \mathrm{~cm}$ for flail leaflet

regurgitant jet. Regurgitant volume and ERO were obtained using the standard formulas (proximal isovelocity surface area method, as recommended by the guidelines). We interpreted $\mathrm{VC}$, regurgitant volume and ERO as being nonesevere in case of no, trace or mild MR. LV ejection fraction (EF) was measured preferably by the biplane method of discs [24]. We measured the LV dimensions in the long parasternal view. Left atrial volume was corrected for BSA to assess LAVI. Pulmonary venous flow pattern was evaluated based on the Doppler signal in the right or left upper pulmonary vein. As atrial fibrillation (AF) can blunt forward systolic pulmonary vein flow, the pulmonary venous flow pattern was considered as 'not applicable' in patients with AF. Pulse waved (PW) Doppler was used to obtain the maximum of the E-wave velocity and TVI MV in the apical 4-chamber view (E-wave velocity was not determined in case of AF). The TVI LVOT was calculated using the corresponding PW signal in the 5-chamber or apical long axis view, with an averaged value in case of AF (eye-balling). This averaged value was based on at least 3 beats. We calculated the right ventricular systolic pressure using the modified Bernoulli 
equation on the transtricuspid continuous-wave Doppler signal, adding the estimated right atrial pressure. The severity of valvular diseases was graded $0-4$ (none $=0$, mild $=1$, moderate $=2$, moderate-severe $=3$, or severe $=4$ ) according to current guidelines, including a definition grade 0.5 for trace MR. In addition, we calculated the change in MR grade between pre- and 1 year post-TAVR. MR severity of grade $\geq 2$ was considered as being significant.

Next, the mitral RegurgitatiOn Severity grading by an Easy-to-use index (ROSE-index) was determined [25]. The ROSE-index is a semiquantitative approach to determine MR severity using 5 instead of 11 recommended echo variables: $R O S E$-index score $=($ valve morphology $* 1)+($ jet char acteristics $* 2)+($ vena contracta $* 2)+($ systolic reversal $* 2)$ $+(L V$ dimensions $* 1)$. Cut-off values of the five parameters (Table 1) were used to score each parameter negative $(0)$ or positive (1) for severe MR. Each score per variable needs to be multiplied by the contribution of that variable ( 1 or 2 in the formula). By adding up the five scores, the total ROSEindex is calculated. In the formula of the ROSE-index, the * symbol stands for multiplication, and values 1 and 2 for the contribution per variable. Total ROSE-index score 0 and 1 indicate moderate MR; score $\geq 4$ is related to severe MR. Patients with index score 2 or 3 need evaluation of the MR severity in a heart valve team or using additional diagnostics. This practical tool showed reliable and reproducible assessment of severe MR (cut-off $\geq 4$ ) in a retrospective analysis of 145 patients with moderate or severe MR. Expert panel consensus reading was considered as the reference standard [25].

\section{Statistical analysis}

Statistical analysis was done using SPSS (version 21.0, IBM Corporation, New York). Continuous variables were expressed as mean $( \pm \mathrm{SD})$ and compared using Student's $t$ test in case of normally distributed data. In case of data that was not normally distribution, we used median [interquartile range (IQR)] and compared data using Wilcoxon signed rank test or Mann Whitney. Categorical data were described using frequencies and percentages, with comparative evaluations performed with the $\chi^{2}$ or McNemar's test for binary results, and $\chi^{2}$ or Wilcoxon signed rank test in case of ordinal data. The Kaplan-Meier method was used to calculate long-term survival for the different grades of MR up through to January 2016. The statistical significance between survival curves of patient subgroups was determined by a log-rank test. A P-value of $<0.05$ was considered statistically significant. Logistic regression for change in MR grade (between the pre-TAVR and 1-year post-TAVR echocardiogram) was performed based on complete case analysis, with univariable and multivariable linear regression on pre-determined variables of interest at baseline (MR grade, MV etiology, TAVR prosthesis type, mitral annulus diameter, LV EF, LAVI, AF and pacemaker (PM) implantation) and potential confounders (age, gender, New York Heart Association (NYHA) class, and TAVR approach).

\section{Results}

\section{Patient characteristics}

Patient and surgical characteristics at baseline are shown in Table 2. Age ranged from 42 to 94 years. All subjects suffered from important AS before intervention, for which 181 Edwards Sapien (85\%) and 32 Corevalve (15\%) prosthesis were successfully implanted. The distribution of TAVR prosthesis type over the etiology subgroups (functional vs. organic MR) was equal. Four patients had a mitral prosthesis in situ before TAVR, and 1 patient underwent MV repair prior to TAVR. MR was present at baseline in $181(85 \%)$ patients. Functional MR ( $45 \%$ of 181 subjects) was secondary to severe AS in 70 patients $(85 \%)$, whereas degenerative annular or valvular calcification was the most frequent cause (92\%) for organic MR (99 patients). MR was absent in the remaining 32 patients before intervention. The etiology at 1-year follow-up remained unchanged (81/182 (45\%) patients with functional MR, 101/182 (55\%) patients with organic MR, and no MR in the remaining 31 patients). Significant mitral stenosis (MS) was only seen in organic MR patients. Forty-six subjects (22\%) showed significant MR (grade $\geq 2$ ). MR grade $\geq 3$ was present in $7.5 \%$ at baseline. Patients with significant MR revealed a higher rate of $\mathrm{AF}$ (30\% vs. $14 \%$ ), PM implantation (13\% vs. $5 \%$ ), TR grade $\geq 2$ ( $28 \%$ vs. $5 \%$ ), aortic regurgitation (AR) grade $\geq 2$ (28\% vs. $13 \%)$ and reduced right ventricular (RV) function ( $24 \%$ vs. 7\%) compared to patients with non-significant MR. Also higher values for mean LAVI, LV dimensions, MV annulus diameter, and SPAP were seen in patients with higher MR grades, whereas the LV EF was lower (45\% vs. 53\%).

\section{Grading of MR related parameters}

In Table 3 the utility of the echo parameters for the semiquantitative approach in patients undergoing TAVR is depicted, expressed as the percentage of patients that was scored per parameter. Overall, there was no difference in the median number of parameters that could be measured pre- or post TAVR ( 9 for both). Jet characteristics were determined in almost all patients, and valve morphology, VC and LAVI in $>90 \%$. The systolic regurgitant flow in the pulmonary veins was difficult to determine due to poor or absent images. Jet characteristics showed the highest positive score for severe MR (73\%), whereas systolic flow 
Table 2 Baseline patient, surgical and echocardiographic characteristics $(n=213)$

\begin{tabular}{|c|c|}
\hline \multicolumn{2}{|l|}{ Patient characteristics } \\
\hline Age, mean years $\pm S D$ & $80.4 \pm 7.3$ \\
\hline Male gender, n (\%) & $94(44.1)$ \\
\hline $\mathrm{BSA}, \mathrm{m}^{2} \pm \mathrm{SD}$ & $1.9 \pm 0.2$ \\
\hline NYHA class $\geq$ III, n (\%) & $105(49.2)$ \\
\hline Pacemaker, n (\%) & $14(6.6)$ \\
\hline \multicolumn{2}{|l|}{ Rhythm, n (\%) } \\
\hline Sinus rhythm & $176(82.6)$ \\
\hline Atrial fibrillation & $37(17.4)$ \\
\hline \multicolumn{2}{|l|}{ Comorbidities, n (\%) } \\
\hline Hypertension & $121(56.8)$ \\
\hline Diabetes & $67(31.5)$ \\
\hline Coronary artery disease & $110(51.6)$ \\
\hline Peripheral artery disease & $38(17.8)$ \\
\hline Prior cerebrovascular accident & $40(20.7)$ \\
\hline Pulmonary disease & $41(19.2)$ \\
\hline Dialysis & $3(1.4)$ \\
\hline Prior cardiac or thoracic intervention & $104(48.8)$ \\
\hline \multicolumn{2}{|l|}{ Surgical characteristics } \\
\hline Logistic EuroSCORE, mean \pm SD & $17.2 \pm 9.3$ \\
\hline \multicolumn{2}{|l|}{ Type of TAVR prosthesis, $\mathrm{n}(\%)$} \\
\hline Edward Sapien & $181(85.0)$ \\
\hline Corevalve & $32(15.0)$ \\
\hline \multicolumn{2}{|l|}{ Type of TAVR access, n (\%) } \\
\hline Transfemoral, n (\%) & $186(87.3)$ \\
\hline Transapical, n (\%) & $23(10.8)$ \\
\hline \multicolumn{2}{|l|}{ Echocardiographic characteristics } \\
\hline ROSE-index score, median (IQR) & $0.0(0.0-0.5)$ \\
\hline \multicolumn{2}{|l|}{ MR grade, $\mathrm{n}(\%)$} \\
\hline No $(0)$ & $32(15.0)$ \\
\hline Trace $(0.5)$ or mild $(1)$ & $135(63.4)$ \\
\hline Moderate (2) & $30(14.1)$ \\
\hline Moderate-severe (3) or severe (4) & $16(7.5)$ \\
\hline \multicolumn{2}{|l|}{ MV etiology in case of MR, $n(\%)(n=181)$} \\
\hline Organic & $99(54.7)$ \\
\hline Functional & $82(45.3)$ \\
\hline Mitral stenosis $\geq$ moderate, $\mathrm{n}(\%)$ & $10(4.7)$ \\
\hline Aortic regurgitation $\geq$ moderate, $\mathrm{n}(\%)$ & $34(16.0)$ \\
\hline Tricuspid regurgitation $\geq$ moderate, $\mathrm{n}(\%)$ & $22(10.3)$ \\
\hline Aortic peak gradient, mean \pm SD $(n=212)$ & $65.8 \pm 21.1$ \\
\hline Aortic mean gradient, mean $\pm \mathrm{SD}(\mathrm{n}=211)$ & $39.1 \pm 14.1$ \\
\hline \multicolumn{2}{|c|}{ Other echocardiographic characteristics, mean \pm SD } \\
\hline SPAP $(\mathrm{mmHg})(\mathrm{n}=130)$ & $40.4 \pm 15.0$ \\
\hline LAVI $\left(\mathrm{ml} / \mathrm{m}^{2}\right)(\mathrm{n}=208)$ & $40.2 \pm 14.6$ \\
\hline Left ventricular EF $(\%)(n=212)$ & $51.6 \pm 12.6$ \\
\hline
\end{tabular}

BSA body surface area, NYHA New York Heart Association, TAVR transcatheter aortic valve replacement, $M R$ mitral regurgitation, $M V$ mitral valve, SPAP systolic artery pressure, $L A V I$ left atrial volume indexed, $E F$ ejection fraction
Table 3 European guideline parameters scored in patients before TAVR $(n=213)$ and 1 year after TAVR $(n=213)$

\begin{tabular}{|c|c|c|c|}
\hline \multirow[t]{2}{*}{ Parameter } & \multicolumn{3}{|c|}{$\%$ of patients scored per parameter } \\
\hline & Pre-TAVR & Post-TAVR & P-value \\
\hline 1. Valve morphology & 97.7 & 94.8 & 0.109 \\
\hline 2. Jet characteristics & 100.0 & 99.5 & 1.000 \\
\hline 3. $V C^{a}$ & 94.8 & 94.8 & 1.000 \\
\hline 4. $\mathrm{ERO}^{\mathrm{a}}$ & 77.9 & 78.4 & 1.000 \\
\hline 5. Regurgitant volume ${ }^{\mathrm{a}}$ & 65.7 & 67.6 & 0.603 \\
\hline \multicolumn{4}{|l|}{ MV inflow pattern } \\
\hline 6. TVI MV/TVI LVOT ratio & 81.2 & 77.0 & 0.314 \\
\hline 7. E-wave & 66.2 & 62.0 & 0.336 \\
\hline 8. SPAP & 62.0 & 49.3 & 0.005 \\
\hline $\begin{array}{l}\text { 9. Systolic regurgitant flow in } \\
\text { pulmonary veins }\end{array}$ & 23.0 & 19.2 & 0.350 \\
\hline 10. LAVI & 97.7 & 94.8 & 0.180 \\
\hline 11. $L V$ dimensions & 83.1 & 80.8 & 0.522 \\
\hline
\end{tabular}

$T A V R$ transcatheter aortic valve replacement, $V C$ vena contracta, $E R O$ effective regurgitant orifice, $M V$ mitral valve, $T V I$ time-velocity integral, LVOT left ventricular outflow tract, SPAP systolic pulmonary artery pressure, $L A V I$ left atrial volume index, $L V$ left ventricular

${ }^{a}$ Including expert interpretation in case of no, trace or mild MR

The parameters (italics) were also used in the calculation of the ROSE-index score

reversal in the pulmonary veins showed a $100 \%$ negative score for MR grade $<3$.

\section{Echocardiographic results}

Overall echo results at follow-up are shown in Table 4. MR was present at 1-year follow-up in $182(85 \%)$ patients. The prevalence of significant AR, aortic gradients and SPAP values were significantly reduced after TAVR. Figure 2 a shows the pre- and post-procedural MR severity, which was not significantly different. In addition, Fig. $2 \mathrm{~b}$ reveals that changes in MR did differ $(\mathrm{P}<0.0001)$ depending on baseline $\mathrm{MR}$ grade. In patients with no MR before TAVR, MR remained absent (37\%), or increased towards trace or mild severity (63\%) at 1-year follow-up. Contrary, in subjects with moderate MR at baseline, the MR grade decreased in more than 3 out of 4 patients $(76 \%)$ after TAVR. The same beneficial effect of unchanged or reduced MR grade following TAVR was seen in patients with moderate-severe and severe MR at baseline. When comparing significant and non-significant MR grade before TAVR, true regression of MR severity after TAVR was seen in 63 and $11 \%$ respectively, indicating a trend towards more reduction for higher MR grade before TAVR.

Overall, significant change in MR was limited: MR grade reduced in 47 patients (22\%) (including a reduction of $>1$ grade in $2 \%$ ), did not change in 130 patients $(61 \%)$, 
Table 4 Clinical and echocardiographic characteristics at 1 year follow-up $(\mathrm{n}=213)$

\begin{tabular}{ll}
\hline Clinical characteristics & \\
Early outcome & \\
New permanent pacemaker, $\mathrm{n}(\%)$ & $18(8.5)$ \\
Tamponade, $\mathrm{n}(\%)$ & $4(1.9)$ \\
Myocardial infarction, $\mathrm{n}(\%)$ & $0(0)$ \\
Cerebrovascular accident, $\mathrm{n}(\%)$ & $6(2.8)$ \\
Late outcome (cumulative) & \\
Post-TAVR, mean years $\pm \mathrm{SD}$ & $1.0 \pm 0.1$ \\
NYHA class $\geq$ III, $\mathrm{n}(\%)$ & $15(7.0)$ \\
New permanent pacemaker, $\mathrm{n}(\%)$ & $23(10.8)$ \\
Atrial fibrillation, $\mathrm{n}(\%)$ & $45(21.1)$ \\
Re-hospitalization, $\mathrm{n}(\%)$ & $57(26.8)$ \\
Aortic valve related & $0(0)$ \\
Due to CHF & $14(6.6)$ \\
Re-intervention, $\mathrm{n}(\%)$ & $0(0)$ \\
Myocardial infarction, $\mathrm{n}(\%)$ & $5(2.3)$ \\
Cerebrovascular accident, $\mathrm{n}(\%)$ & $10(4.7)$ \\
Echocardiographic characteristics & \\
ROSE-index score, median $(\mathrm{IQR})$ & $35.9 \pm 10.6$ \\
MR grade, $\mathrm{n}(\%)$ & $40.9 \pm 16.8$ \\
No (0) & $52.0 \pm 11.1$ \\
Trace $(0.5)$ or mild (1) & $0.0(0.0-0.0)$ \\
Moderate $(2)$ & \\
Moderate-severe $(3)$ or severe $(4)$ & $31(14.6)$ \\
Mitral stenosis $\geq$ moderate, $\mathrm{n}(\%)$ & $149(70.0)$ \\
Aortic regurgitation $\geq$ moderate, $\mathrm{n}(\%)$ & $16(7.5)$ \\
Tricuspid regurgitation $\geq$ moderate, $\mathrm{n}(\%)$ & $17(8.0)$ \\
Aortic peak gradient, mean $\pm \mathrm{SD}$ & $10(4.7)$ \\
Aortic mean gradient, mean $\pm \mathrm{SD}$ & $21(9.9)$ \\
Other echocardiographic characteristics, mean $\pm \mathrm{SD}$ & $26(12.2)$ \\
SPAP (mmHg) & $15.8 \pm 6.7$ \\
LAVI (ml/m $\left.{ }^{2}\right)$ & $8.2 \pm 3.5$ \\
\hline
\end{tabular}

TAVR transcatheter aortic valve replacement, NYHA New York Heart Association, $C H F$ congestive heart failure, $M R$ mitral regurgitation, $S P A P$ systolic artery pressure, $L A V I$ left atrial volume indexed, $E F$ ejection fraction

and increased in 31 patients (17\%) (including an increase of $>1$ grade in $2 \%$ ). Significant MR (grade $\geq 2$ ) at 1 -year follow-up was diagnosed in 33 patients (15\%). This subgroup showed a higher rate of significant TR (33\% vs. $8 \%$ ), median MR change, SPAP and LAVI. In these patients the baseline MR severity had been trace or mild in $42 \%$, and grade $\geq 3$ in $10 \%$. MR grade $\geq 3$ was present in 16 patients (8\%) at 1 year follow-up. In none of these patients the MR had been improved. Regarding the TAVR prosthesis type, equal medians of change in MR grade for both prosthesis $(\mathrm{P}=0.903)$ were seen. As depicted in Fig. 3, medians of
MR grade were also similar between the organic and functional subgroups $(\mathrm{P}=0.918)$.

The median ROSE-index score for patients with nonsevere MR was 0 , compared to a median score of 3 for patients with $M R \geq$ grade $3(\mathrm{P}<0.0001)$. In Online Resource 1 of the Supplementary material the results are depicted of the comparison between the MR grade using 11 parameters from the European guidelines (clinical diagnosis), and the 5-parameter ROSE-index. MR severity was correctly determined by the ROSE-index in 381 of the 426 TTE's (89\%). In 3 echocardiograms $(0.7 \%)$ severe MR was misdiagnosed. A total of 27 patients with MR grade $<3$, and 15 patients with MR grade $\geq 3$ had a ROSE-index score of 2 or 3, meaning that in $10 \%$ of the patients additional diagnostics and/ or evaluation in a heart valve team is recommended [25].

\section{Regression analysis}

Univariable regression analysis (Online Resource 2, Supplementary material) showed a significant, negative correlation between change in MR grade and MR severity and LV EF at baseline $(r=-0.496, P<0.0001$, and $r=-0.011, P=0.004$ respectively). In a multivariable regression model correcting for other parameters and confounders, MR grade at baseline remained independently correlated $(\mathrm{r}=-0.470, \mathrm{P}<0.0001)$, suggesting more improvement of MR grade in patients with higher MR grade before intervention compared to a lower severity. In addition, the variable PM implantation showed a significant positive correlation after multivariable regression analysis $(r=0.533, P=0.006)$, related to less improvement of MR in patients with a PM in situ.

\section{Clinical results}

Clinical outcomes are depicted in Table 4. Overall, NYHA functional class was lower after TAVR. A reduction was seen in $68 \%$, whereas $28 \%$ of the subjects showed no change in symptoms. At 1-year follow-up, patients with $\geq$ moderate MR post-TAVR experienced more symptoms and AF compared to non-significant MR. Ten patients (5\%) with $\mathrm{AF}$ at baseline were converted to sinus rhythm during the follow-up TTE. Of the 45 (21\%) patients with AF at followup, AF de novo was seen in 18 subjects. Regarding the complications at follow-up, cerebrovascular events were most frequently seen (independent of AF). Re-hospitalization or re-intervention resulting from a TAVR prosthesis problem was never seen. In two patients a Mitraclip procedure was performed at follow-up: 1 patient showed a reduction of MR but in both cases symptoms did not improve.

As we only included patients with TTE at baseline and 1-year follow-up, no early or 1 year mortality rates were calculated (all patients were alive at 1 year). The overall survival at $2.7 \pm 1.2$ years after TAVR was $80.3 \%$. Although 
a trend was seen for higher mortality in patients with MR grade $\geq 2$, Fig. 4 shows no significant difference in survival based on the MR grade after TAVR $(P=0.097)$. Neither was there a significant difference in survival rates depending on pre-TAVR MR grades, or improvement of MR at follow-up. Twenty-three patients (11\%) died of a cardiovascular related cause (including 14 patients with unknown cause). In the subgroup of patients with significant MR, 11 (33\%) died (cardiovascular cause in six patients, including $2 \times$ heart failure and $4 \times$ unknown cause). The mortality number amongst patients with less than moderate MR was $31(17 \%)$ (cardiovascular cause in 17 patients, including $4 \times$ heart failure, $1 \times$ out-of-hospital-cardiac-arrest, $1 \times$ cerebrovascular event, and $11 \times$ unknown cause).

\section{Discussion}

Our study revealed four important findings: First, the systematic 2D echocardiographic approach used in this study for MR grading was feasible in both pre- and post-TAVR echocardiograms. The practical scoring index developed by our group (ROSE-index) using only five recommended parameters, correctly excluded severe MR in almost all subjects. Secondly, significant MR was seen in $22 \%$ before and in $15 \%$ at 1 year after TAVR. Thirdly, severity did not change in most patients, although regression analysis revealed that higher MR grade and absence of PM implantation at baseline was related to more MR improvement after TAVR. Lastly, overall survival was not significantly influenced by the MR grade 1-year post-TAVR, although a trend for higher mortality was seen in patients with significant MR.

\section{Grading methods}

Previous data regarding the prevalence of incidental MR in severe AS varies widely, depending on the study inclusion criteria, echocardiographic definitions for MR, and diverse non-standardized grading methods $[5,13,14,26,27]$. Single qualitative methods have been used $[7,8,10,12,16-18,28$, 29], although for more accurate grading current guidelines recommend the integration of various qualitative and quantitative echo measurements [20-22]. However, systematic quantitative assessments are rarely applied in routine evaluation due to the time consuming aspect and technical limitations [6, 30-33]. Imaging tools like 3D echocardiography and multidetector computed tomography might in the future overcome these issues. Nowadays their routine use is still limited [6, 34].

Our data showed that standardized MR grading was feasible and resulted in the assessment of almost all guideline parameters. In accordance with previous studies, color flow imaging was highly sensitive [31]. The VC showed a high specificity, confirming to be less susceptible to physiologic loading conditions [27]. The systolic flow reversal of the pulmonary veins was relevant based on its specificity [36], however, it was sparsely available in short-focused TTE's. Ideal would be a "scoring system" for accurate and reproducible MR grading in both pre- and post-TAVR echocardiograms. We proposed the 5-parameter ROSE-index, to aid the treating physician in how to use the integrated approach as recommended by the guidelines for the determination of MR severity in patients undergoing TAVR [25]. Although the index was originally developed to distinguish moderate from severe MR in clinical practice, it correctly determined MR severity in $89 \%$ of our study patients. The ROSE-index showed a high specificity and negative predictive value for severe MR, which is preferable to exclude severe MR in these patients, thereby preventing unwarranted double-valve intervention or surgical refusal. The reliable and reproducible assessment of severe MR (cut-off $\geq 4$ ) by this practical index will not only be helpful for clinical decision making, it will also be of additional value for follow-up and research purposes.

\section{Prevalence of significant MR}

The majority of patients in our series had some degree of MR at baseline (85\%), similar to previous studies revealing MR in $60-90 \%$ of TAVR patients [5, 16, 35]. Moderate MR has been reported in up 13-17\% in the studies of Barreiro et al. and Ruel et al. [14, 26], 20 and 22\% in both PARTNER trial cohorts $[7,10]$, and $33 \%$ in the study of Vollenbroich et al. including $>1000$ patients [36], which was in line with our data. Overall rate of MR grade $>3$ was also low $(<10 \%)$ in a previously performed meta-analysis and therefore similar to our results [37]. The higher prevalence of an organic etiology in the current study, as also seen in previous studies $[6,16,38]$, may be explained by the relatively high-aged patients with multiple comorbidities that undergo TAVR. These patients are prone to develop sclerotic calcification of the mitral apparatus ( $92 \%$ in our study).

\section{Change in MR grade}

In line with current literature [16, 39], our data showed that there was no change in MR grade amongst most patients (61\%) at 1-year follow-up. Seventy-eight percent of the TAVR patients had no MR or less than moderate MR at baseline. It is known that these patients show little regression or progression after TAVR [40, 41]. On the other hand our study showed more improvement in subjects with higher MR grade before intervention (63\%). The same effect was seen in the studies of Tzikas et al. and Almasood et al. [12, 42], and confirmed in our multivariable regression analysis for change in MR. Furthermore, 
A
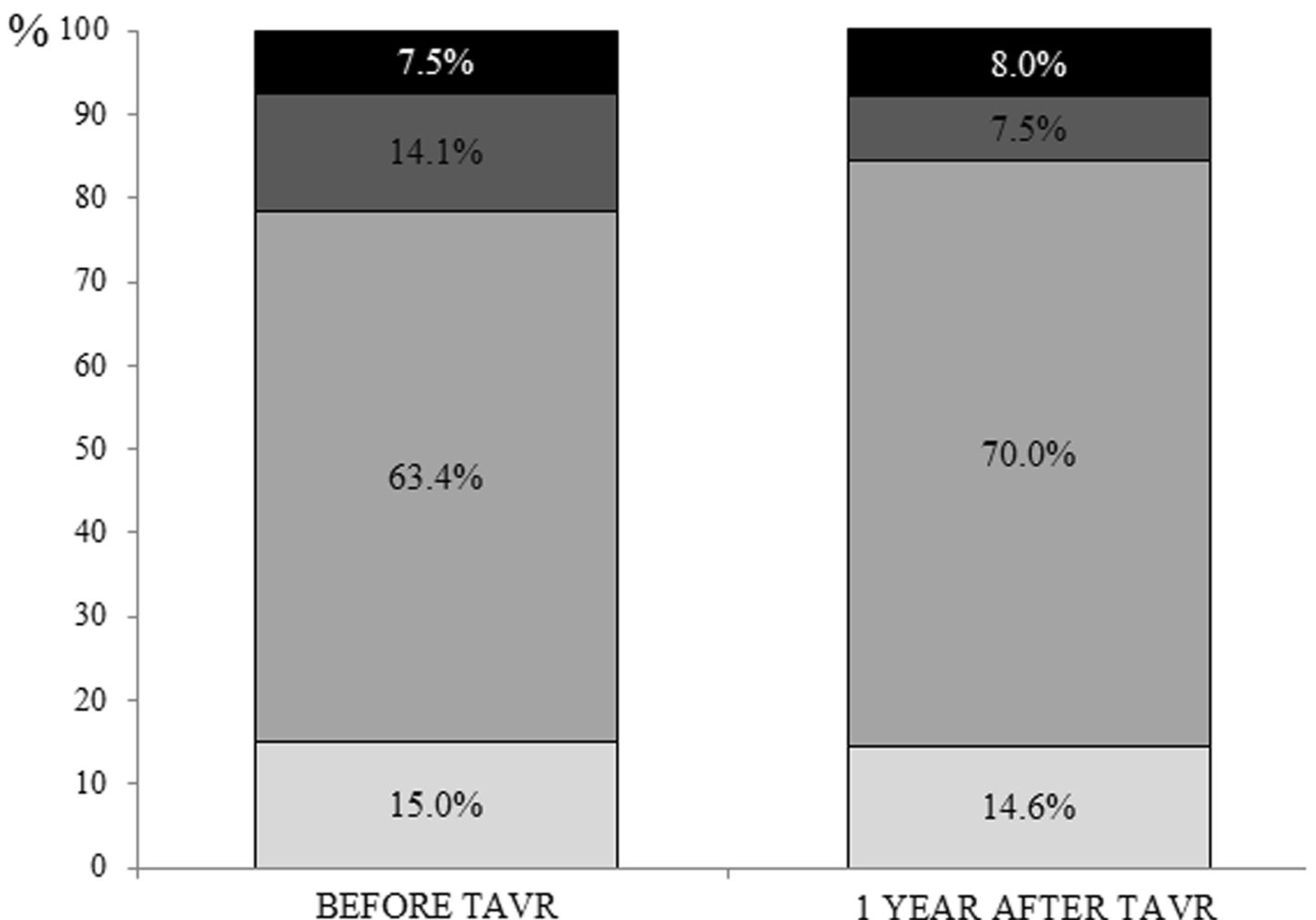

BEFORE TAVR

1 YEAR AFTER TAVR

moderate-severe or severe mitral regurgitation

$\square$ moderate mitral regurgitation

$\square$ trace or mild mitral regurgitation

$\square$ no mitral regurgitation

B

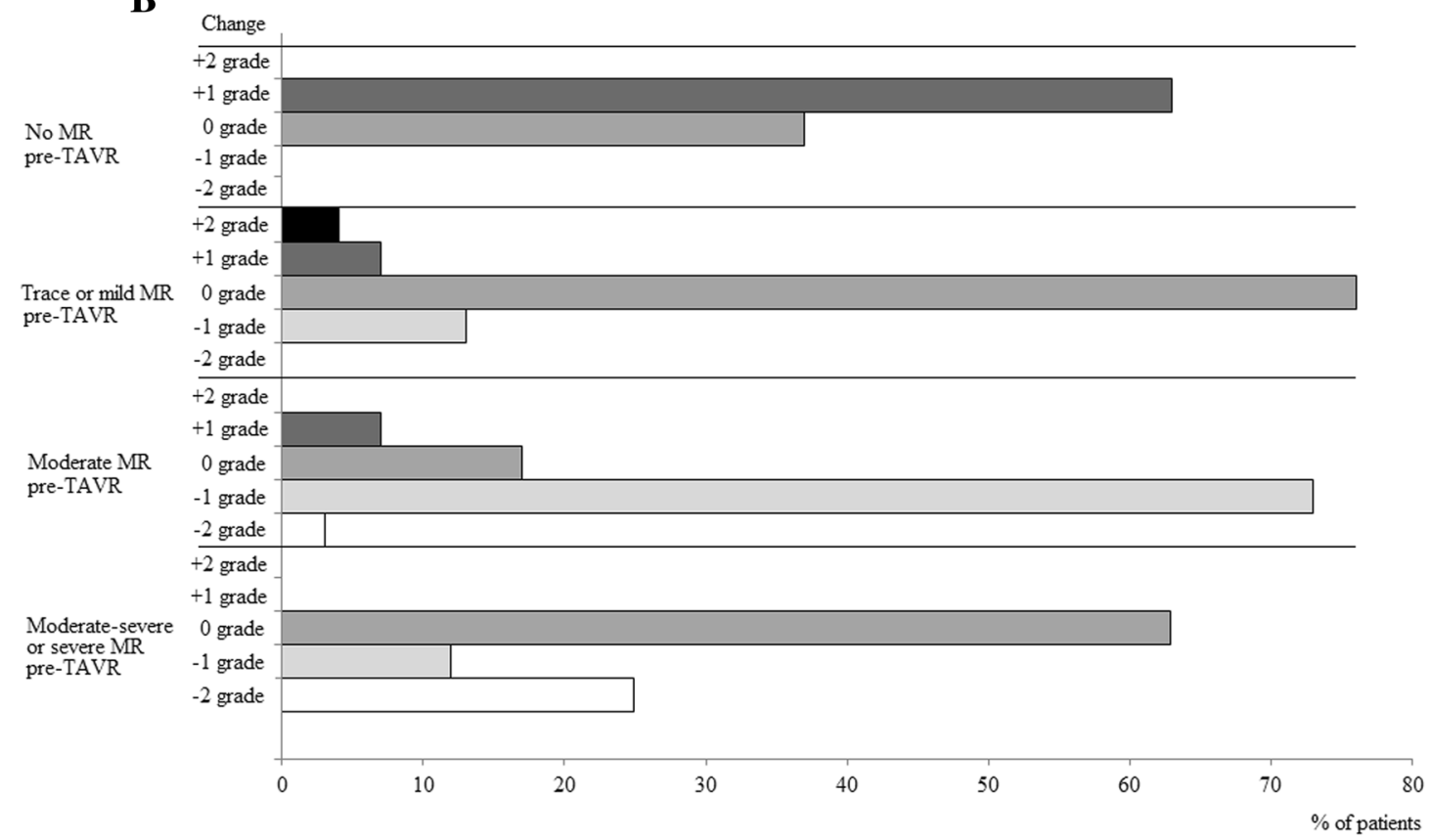


4Fig. 2 MR grade (a) and change in severity per pre-TAVR MR grade (b) in patients undergoing TAVR with both baseline and 1-year follow-up TTE available $(n=213)$. a Median (IQR) grade of MR at baseline: $1.0(1.0-1.0)$, and 1 year after TAVR: $1.0(1.0-1.0)$, $\mathrm{P}=0.311$. b Median (IQR) change in patients at baseline with no: $1.0(0.0-1.0)$, mild or trace: $0.0(0.0-0.0)$, moderate $-1.0(-1.0$ to -0.8$)$, and moderate-severe or severe MR: 0.0 ( -1.8 to 0.0$)$, $\mathrm{P}<0.0001$

a trend for more improvement in patients with functional etiology was not seen, which was to be expected from literature revealing coaptation of the otherwise normal MV leaflets, and persistent restriction of leaflet mobility in organic (calcified) MR [11, 18]. This similar change for both etiologies was also seen in the studies of Tzikas et al., Unger et al. and Almasood et al. [12, 15, 42] Moreover, previous studies showed in particular an improvement in MR depending on the implanted prosthesis type $[8,12$, 19, 35].Mainly self-expandable valves seem to be at risk for deep implants, resulting in the valve cage impinging on the anterior MV leaflet, and thereby increasing MR [12]. Nevertheless, this correlation was not observed in our study. Further analysis of any valve malpositioning in our study showed malpositioning after TAVR in only six patients (2.8\%), and revealed no significant correlation between prosthesis malpositioning and change in MR grade $(r=0.053, P=0.861)$. In addition, the size of the chosen prosthesis might play a role in the incidence of patient-prosthesis mismatch, which is overall less common in patients after TAVR, nevertheless may limit decrease of MR due to persistently high LV pressures. So far, no studies have directly compared TAVR in terms of MR reduction related to the presence of patient-prosthesis mismatch. Although we did not collect any data regarding the aortic EOA after TAVR (mainly used to determine patient-prosthesis mismatch) [43] we were able to perform univariable analyses for change of MR and (1) prosthesis valve size, and (2) prosthesis valve size index (indexed for body surface area). The correlation between valve size and change in MR was not significant $(\mathrm{r}=-0.022, \mathrm{P}=0.915)$, nor was the correlation between valve size indexed and change in MR $(r=-0.124, P=0.679)$. Third, calcified AS affects mitro-aortic anatomical configuration and mitroaortic coupling, possibly leading to the persistence of MR after TAVR. Moreover, calcification of the mitro-aortic junction seems related to a higher likelihood of left bundle branch block, inducing less reduction of MR post TAVR [44]. In 50\% of the patients with MR before TAVR in our study, the etiology of MR was based on calcification.
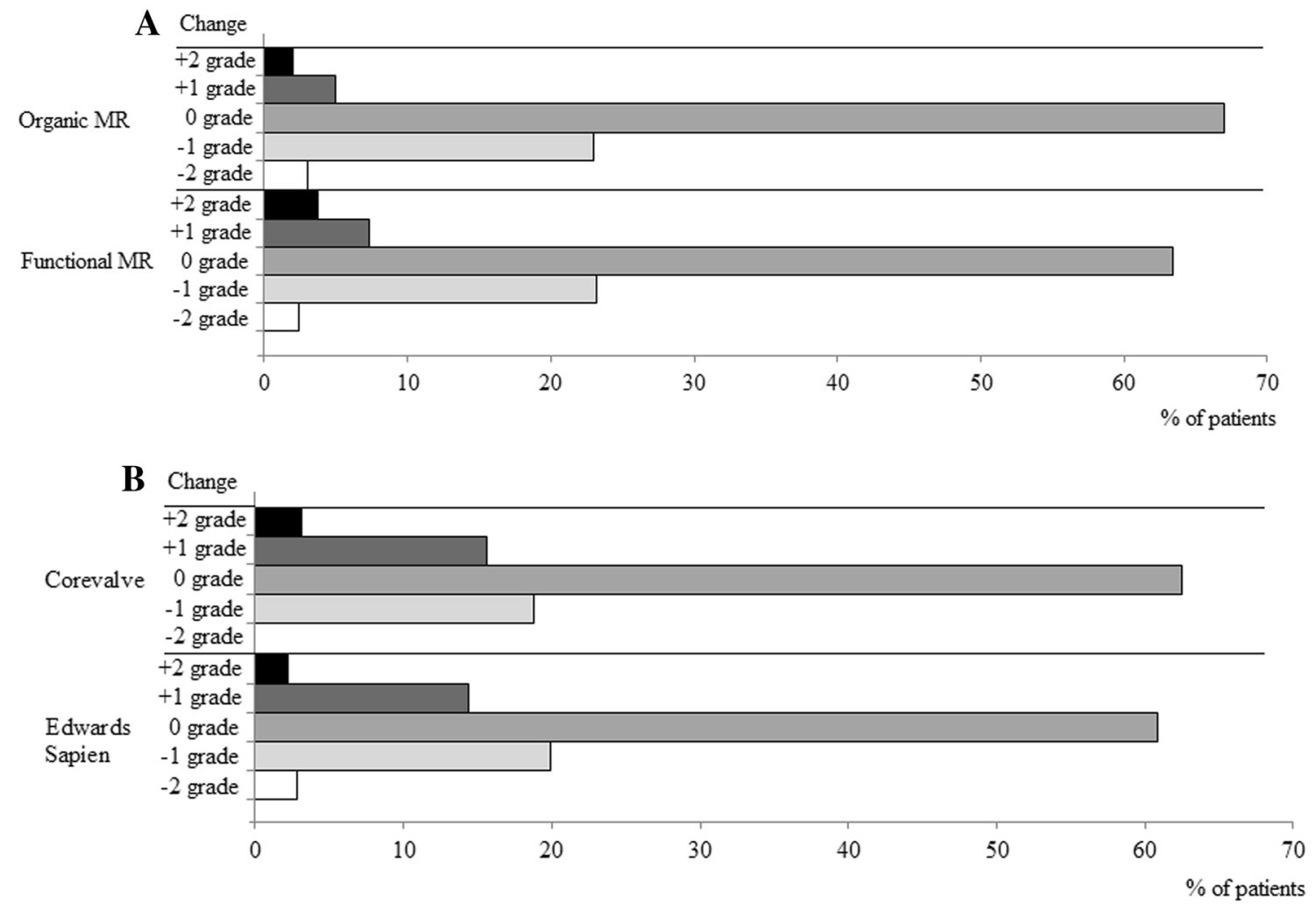

Fig. 3 Change in grade of MR per MV etiology (a) and type of TAVR prosthesis (b). a Median (IQR) change in functional: 0.0 $(-1.0$ to 0.0$)$ and organic MR: $0.0(-1.0$ to 0.0$)$. $\mathrm{P}=0.918$. b Median
(IQR) change in Edwards Sapien: $0.0(0.0-0.0)$ and Corevalve prosthesis: $0.0(0.0-0.0), \mathrm{P}=0.903$ 
Unfortunately, we were not able to retrospectively determine the exact location of the calcified parts. Lastly a pacemaker in situ was correlated to less improvement of MR after TAVR, which may be explained by the fact that RV apical pacing significantly induces MR [45].

\section{Clinical outcome}

Our data revealed no significant difference in long-term survival depending on MR grade at 1 year after TAVR. Same results were found in other studies [8, 46, 47]. Preexisting physical conditions such as AF, poor LV function, high LV volumes, and large LAVI (particularly seen in patients with MR grade $\geq 2$ ) [8, 11, 16] may contribute to higher mortality rates observed in the short-term [14, 29, 46]. However, after 1-year follow-up a regression of concentric myocardial hypertrophy due to a decrease in ventricular afterload, and/or reverse remodeling that lead to changes in LV shape and geometry, may influence MV hemodynamics and therefore these confounding variables $[11,12,16,18,19,35]$. A second explanation is that the clinical profiles of patients with MR grade $\geq 2$ and $<2$ were similar in our study. Thus significantly higher mortality caused by comorbidities was not seen. However, we did observe a trend towards lower survival rates for at least moderate MR, in accordance with the FRANCE 2 and Spanish TAVR registries [47].

Additional subanalyses regarding the mortality rates according to pre-TAVR MR grades and MR improvement also showed no significant differences, which is in line with other series $[8,9,11,47]$. This finding is opposite to surgical aortic valve replacement, where concomitant moderate MR has demonstrated to be an independent risk factor for long-term mortality [14]. It might be that a higher early, but not late, mortality rate was present in our patients with grade $\geq 2 \mathrm{MR}$ [11].

Lastly, clinical outcome measure (NYHA functional class) showed an improvement in almost 2 out of 3 patients regardless the pre-TAVR MR grade, as seen before [11, 35]. This improvement of symptoms despite significant $\mathrm{MR}$, is an important consideration for the fragile, mainly elderly patients that undergo TAVR.

\section{Limitations}

This study was retrospective with its inherent limitations. Evaluation of 2D TTE in daily practice may be limited by suboptimal imaging windows, especially in short-focused examinations. The TAVR procedures were performed in a
Fig. 4 Kaplan-Meier to illustrate survival depending on grade of MR starting at 1 year after TAVR $(\mathrm{n}=213)$

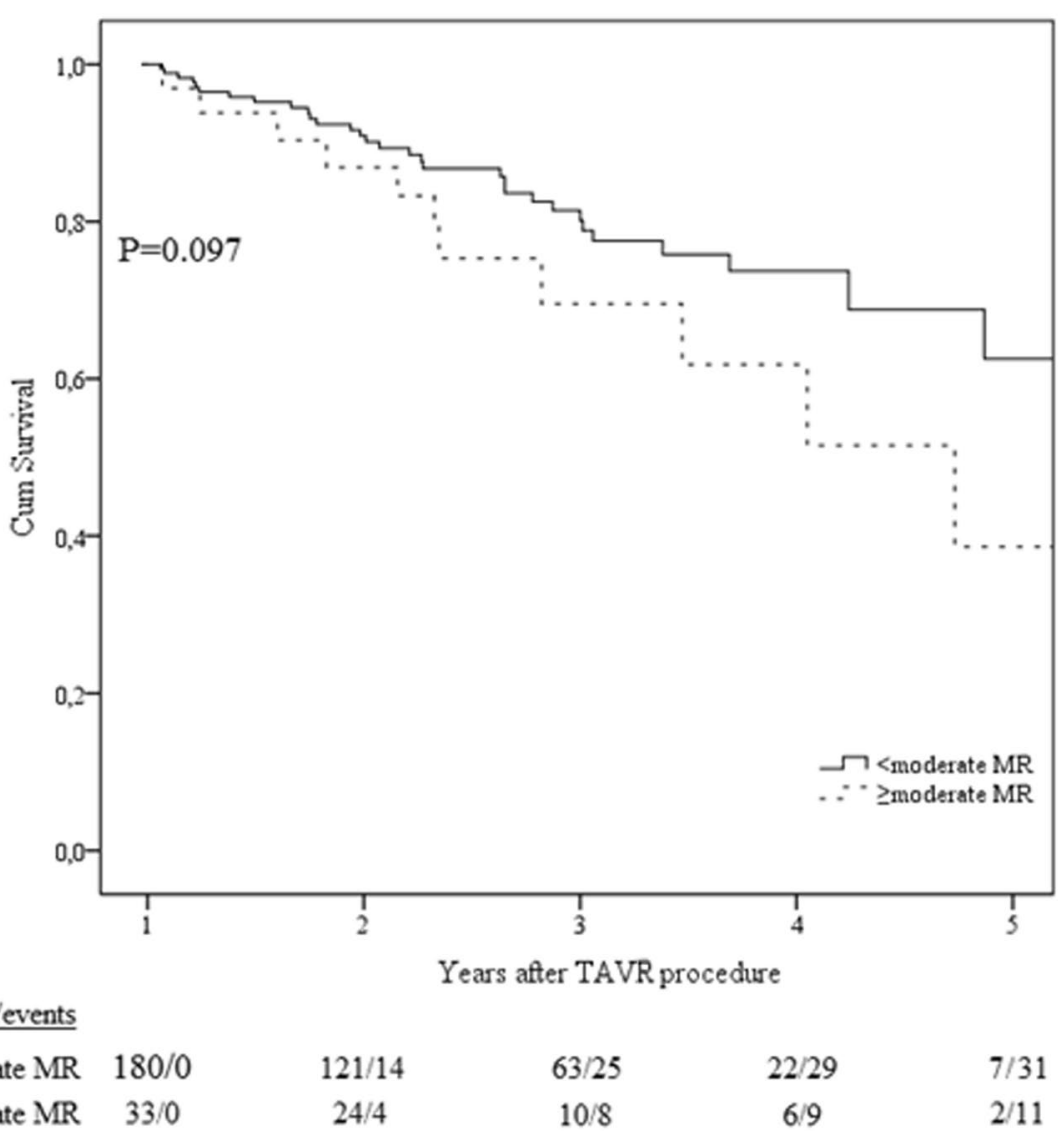


single academic center. Echocardiographic follow-up was not performed in all subjects undergoing TAVR, and there was no screening $\log$ of patients that underwent surgical aortic valve replacement or conservative management, potentially leading to a selection bias. As there were only a few subjects with more than moderate MR, we were not able to perform any analyses on the endpoint grade $\geq 3 \mathrm{MR}$ at 1 year follow-up.

\section{Conclusion}

Grading of MR by an systematic 2D echocardiographic approach in patients undergoing TAVR revealed that most patients had some degree of MR, which was less than moderate and seldom severe in most patients. Overall there was no change in the MR grade comparing pre-TAVR and 1 year follow-up TTE's, although higher baseline MR grades showed more reduction and lower grades revealed more increase of severity, but never leading to significant MR. The present series showed a positive effect of TAVR on outcome regardless MR severity, demonstrated by similar long-term survival and NYHA class improvement amongst post-TAVR MR grades. Based on our data, we propose no concomitant MV intervention in this specific cohort. Clinical decision making can be safely based on the systematic evaluation of MR severity by an integrated 2D echo approach.

\section{Compliance with ethical standards}

Conflict of interest The authors declare that they have no conflict of interest.

Open Access This article is distributed under the terms of the Creative Commons Attribution 4.0 International License (http://creativeco mmons.org/licenses/by/4.0/), which permits unrestricted use, distribution, and reproduction in any medium, provided you give appropriate credit to the original author(s) and the source, provide a link to the Creative Commons license, and indicate if changes were made.

\section{References}

1. Iung B, Baron G, Butchart EG, Delahaye F, Gohlke-Barwolf C, Levang OW et al (2003) A prospective survey of patients with valvular heart disease in Europe: The Euro Heart Survey on Valvular Heart Disease. Eur Heart J 24:1231-1243

2. Nkomo VT, Gardin JM, Skelton TN, Gottdiener JS, Scott CG, Enriquez-Sarano M (2006) Burden of valvular heart diseases: a population-based study. Lancet 368:1005-1011

3. Osnabrugge RL, Mylotte D, Head SJ, Van Mieghem NM, Nkomo VT, LeReun CM et al (2013) Aortic stenosis in the elderly: disease prevalence and number of candidates for transcatheter aortic valve replacement: a meta-analysis and modeling study. J Am Coll Cardiol 62:1002-1012
4. Mylotte D, Osnabrugge RL, Windecker S, Lefevre T, de Jaegere $P$, Jeger R et al (2013) Transcatheter aortic valve replacement in Europe: adoption trends and factors influencing device utilization. J Am Coll Cardiol 62:210-219

5. Unger P, Dedobbeleer C, Van CG, Plein D, Cosyns B, Lancellotti P (2010) Mitral regurgitation in patients with aortic stenosis undergoing valve replacement. Heart 96:9-14

6. Nombela-Franco L, Ribeiro HB, Urena M, Allende R, AmatSantos I, DeLarochelliere R et al (2014) Significant mitral regurgitation left untreated at the time of aortic valve replacement: a comprehensive review of a frequent entity in the transcatheter aortic valve replacement era. J Am Coll Cardiol 63:2643-2658

7. Leon MB, Smith CR, Mack M, Miller DC, Moses JW, Svensson LG et al (2010) Transcatheter aortic-valve implantation for aortic stenosis in patients who cannot undergo surgery. N Engl J Med 363:1597-1607

8. Bedogni F, Latib A, De MF, Agnifili M, Oreglia J, Pizzocri S et al (2013) Interplay between mitral regurgitation and transcatheter aortic valve replacement with the CoreValve Revalving System: a multicenter registry. Circulation 128:2145-2153

9. Hutter A, Bleiziffer S, Richter V, Opitz A, Hettich I, Mazzitelli D et al (2013) Transcatheter aortic valve implantation in patients with concomitant mitral and tricuspid regurgitation. Ann Thorac Surg 95:77-84

10. Smith CR, Leon MB, Mack MJ, Miller DC, Moses JW, Svensson LG et al (2011) Transcatheter versus surgical aortic-valve replacement in high-risk patients. N Engl J Med 364:2187-2198

11. Toggweiler S, Boone RH, Rodes-Cabau J, Humphries KH, Lee M, Nombela-Franco L et al (2012) Transcatheter aortic valve replacement: outcomes of patients with moderate or severe mitral regurgitation. J Am Coll Cardiol 59:2068-2074

12. Tzikas A, Piazza N, van Dalen BM, Schultz C, Geleijnse ML, van Geuns RJ et al (2010) Changes in mitral regurgitation after transcatheter aortic valve implantation. Cathet Cardiovasc Interv 75:43-49

13. Caballero-Borrego J, Gomez-Doblas JJ, Cabrera-Bueno F, Garcia-Pinilla JM, Melero JM, Porras C et al (2008) Incidence, associated factors and evolution of non-severe functional mitral regurgitation in patients with severe aortic stenosis undergoing aortic valve replacement. Eur J Cardiothorac Surg 4:62-66

14. Barreiro CJ, Patel ND, Fitton TP, Williams JA, Bonde PN, Chan $\mathrm{V}$ et al (2005) Aortic valve replacement and concomitant mitral valve regurgitation in the elderly: impact on survival and functional outcome. Circulation 112:I443-I447

15. Unger P, Plein D, Van CG, Cosyns B, Pasquet A, Henrard V et al (2008) Effects of valve replacement for aortic stenosis on mitral regurgitation. Am J Cardiol 102:1378-1382

16. Hekimian G, Detaint D, Messika-Zeitoun D, Attias D, Iung B, Himbert D et al (2012) Mitral regurgitation in patients referred for transcatheter aortic valve implantation using the Edwards Sapien prosthesis: mechanisms and early postprocedural changes. J Am Soc Echocardiogr 25:160-165

17. Giordana F, Capriolo M, Frea S, Marra WG, Giorgi M, Bergamasco L et al (2013) Impact of TAVI on mitral regurgitation: a prospective echocardiographic study. Echocardiography 30:250-257

18. Durst R, Avelar E, McCarty D, Poh KK, Friera LF, Llano MF et al (2011) Outcome and improvement predictors of mitral regurgitation after transcatheter aortic valve implantation. J Heart Valve Dis 20:272-281

19. Samim M, Stella PR, Agostoni P, Kluin J, Ramjankhan F, Sieswerda G et al (2011) Transcatheter aortic implantation of the Edwards-SAPIEN bioprosthesis: insights on early benefit of TAVI on mitral regurgitation. Int J Cardiol 152:124-126 
20. Nishimura RA, Otto CM, Bonow RO, Carabello BA, Erwin JP III, Fleisher LA et al (2017) 2017 AHA/ACC focused update of the 2014 AHA/ACC guideline for the management of patients with valvular heart disease: A Report of the American College of Cardiology/American Heart Association Task Force on Clinical Practice Guidelines. J Am Coll Cardiol 70:252-289

21. Baumgartner H, Falk V, Bax JJ, De Bonis M, Hamm C, Holm PJ et al (2017) 2017 ESC/EACTS Guidelines for the management of valvular heart disease. Eur Heart J 38:2739-2791

22. Zoghbi WA, Enriquez-Sarano M, Foster E, Grayburn PA, Kraft CD, Levine RA et al (2003) Recommendations for evaluation of the severity of native valvular regurgitation with two-dimensional and Doppler echocardiography. J Am Soc Echocardiogr 16:777-802

23. Lancellotti P, Tribouilloy C, Hagendorff A, Popescu BA, Edvardsen T, Pierard LA et al (2013) Recommendations for the echocardiographic assessment of native valvular regurgitation: an executive summary from the European Association of Cardiovascular Imaging. Eur Heart J Cardiovasc Imaging 14:611-644

24. Lang RM, Badano LP, Mor-Avi V, Afilalo J, Armstrong A, Ernande L et al (2015) Recommendations for cardiac chamber quantification by echocardiography in adults: an update from the American Society of Echocardiography and the European Association of Cardiovascular Imaging. Eur Heart J Cardiovasc Imaging 16:233-270

25. Jansen R, Hart EA, Peters M, Urgel K, Kluin J, Tietge WJ et al (2017) An easy-to-use scoring index to determine severity of mitral regurgitation by $2 \mathrm{D}$ echocardiography in clinical practice. Echocardiography 34:1275-1283

26. Ruel M, Kapila V, Price J, Kulik A, Burwash IG, Mesana TG (2006) Natural history and predictors of outcome in patients with concomitant functional mitral regurgitation at the time of aortic valve replacement. Circulation 114:I541-I546

27. Waisbren EC, Stevens LM, Avery EG, Picard MH, Vlahakes GJ, Agnihotri AK (2008) Changes in mitral regurgitation after replacement of the stenotic aortic valve. Ann Thorac Surg 86:56-62

28. Gilard M, Eltchaninoff H, Iung B, Donzeau-Gouge P, Chevreul K, Fajadet J et al (2012) Registry of transcatheter aortic-valve implantation in high-risk patients. N Engl J Med 366:1705-1715

29. Di MC, Eltchaninoff H, Moat N, Goicolea J, Ussia GP, Kala P et al (2013) The 2011-12 pilot European Sentinel Registry of Transcatheter Aortic Valve Implantation: in-hospital results in 4,571 patients. EuroIntervention 8:1362-1371

30. Thavendiranathan P, Phelan D, Collier P, Thomas JD, Flamm SD, Marwick TH (2012) Quantitative assessment of mitral regurgitation: how best to do it. JACC Cardiovasc Imaging 5:1161-1175

31. Biner S, Rafique A, Rafii F, Tolstrup K, Noorani O, Shiota T et al (2010) Reproducibility of proximal isovelocity surface area, vena contracta, and regurgitant jet area for assessment of mitral regurgitation severity. JACC Cardiovasc Imaging 3:235-243

32. Enriquez-Sarano M, Tribouilloy C (2002) Quantitation of mitral regurgitation: rationale, approach, and interpretation in clinical practice. Heart 88:iv1-iv3

33. Baumgartner H, Schima H, Kuhn P (1991) Value and limitations of proximal jet dimensions for the quantitation of valvular regurgitation: an in vitro study using Doppler flow imaging. J Am Soc Echocardiogr 4:57-66
34. Amat-Santos IJ, Revilla A, Lopez J, Cortes C, Gutierrez H, Serrador A et al (2015) Value of CT in patients undergoing selfexpandable TAVR to assess outcomes of concomitant mitral regurgitation. JACC Cardiovasc Imaging 8:226-227

35. Webb JG, Pasupati S, Humphries K, Thompson C, Altwegg L, Moss R et al (2007) Percutaneous transarterial aortic valve replacement in selected high-risk patients with aortic stenosis. Circulation 116:755-763

36. Vollenbroich R, Stortecky S, Praz F, Lanz J, Franzone A, Zuk $\mathrm{K}$ et al (2016) The impact of functional vs degenerative mitral regurgitation on clinical outcomes among patients undergoing transcatheter aortic valve implantation. Am Heart J 184:71-80

37. Harling L, Saso S, Jarral OA, Kourliouros A, Kidher E, Athanasiou T (2011) Aortic valve replacement for aortic stenosis in patients with concomitant mitral regurgitation: should the mitral valve be dealt with?. Eur J Cardiothorac Surg 40:1087-1096

38. Kiramijyan S, Koifman E, Asch FM, Magalhaes MA, Didier R, Escarcega RO et al (2016) Impact of functional versus organic baseline mitral regurgitation on short- and long-term outcomes after transcatheter aortic valve replacement. Am J Cardiol 117:839-846

39. Zakkar M, Alassar A, Lopez-Perez M, Roy D, Brecker S, Sharma $R$ et al (2015) Left ventricular remodeling after transcatheter aortic valve implantation: one-year follow-up study. Innovations (Phila) 10:44-47

40. Schubert SA, Yarboro LT, Madala S, Ayunipudi K, Kron IL, Kern JA et al (2016) Natural history of coexistent mitral regurgitation after aortic valve replacement. J Thorac Cardiovasc Surg 151:1032-1039, 1042

41. Kaczorowski DJ, Macarthur JW, Howard J, Kobrin D, Fairman A, Woo YJ (2013) Quantitative evaluation of change in coexistent mitral regurgitation after aortic valve replacement. J Thorac Cardiovasc Surg 145:341-347

42. Almasood A, Al AS, El-Shurafa H, Alotaibi M, Al KS, AlAbdallah $M$ et al (2015) The change in mitral regurgitation severity after trans-catheter aortic valve implantation. J Saudi Heart Assoc 27:10-17

43. Rahimtoola SH (1978) The problem of valve prosthesis-patient mismatch. Circulation 58:20-24

44. Amat-Santos IJ, Revilla A, López J, Cortés C, Gutiérrez H, Serrador A et al (2015) Value of CT in patients undergoing selfexpandable TAVR to assess outcomes of concomitant mitral regurgitation. JACC Cardiovasc Imaging 8:226-231

45. Alizadeh A, Sanati HR, Haji-Karimi M, Yazdi AH, Rad MA, Haghjoo M et al (2011) Induction and aggravation of atrioventricular valve regurgitation in the course of chronic right ventricular apical pacing. Europace 13:1587-1590

46. Kiramijyan S, Magalhaes MA, Koifman E, Didier R, Escarcega $\mathrm{RO}$, Minha $\mathrm{S}$ et al (2016) Impact of baseline mitral regurgitation on short- and long-term outcomes following transcatheter aortic valve replacement. Am Heart J 178:19-27

47. Sabate M, Canovas S, Garcia E, Hernandez AR, Maroto L, Hernandez JM et al (2013) In-hospital and mid-term predictors of mortality after transcatheter aortic valve implantation: data from the TAVI National Registry 2010-2011. Rev Esp Cardiol (Engl Ed) 66:949-958 